\title{
Delayed Cerebellar Ataxia: Neurological sequelae of Malaria in an Adolescent Girl
}

\section{Sabrina Bakeera-Kitaka ${ }^{1,2}$, Andrew Weil Semulimi ${ }^{1}$, Namanya Sylvester ${ }^{1}$, Nestor Mbabazi ${ }^{1}$, Victor Musiime ${ }^{1,2}$, Irene Lubega ${ }^{1}$ and Edison Arwanire Mworozi ${ }^{1 *}$}

${ }^{1}$ Mulago National Referral Hospital Kampala, Uganda

${ }^{2}$ Department of Pediatrics and Child Health, Makerere University College of Health

Sciences, Uganda

*Corresponding Author: Edison Arwanire Mworozi, Mulago National Referral

Hospital Kampala, Uganda.
Received: September 30, 2021

Published: November 23, 2021

(C) All rights are reserved by Edison Arwanire Mworozi., et al.

\begin{abstract}
Background: Malaria remains a serious infectious disease causing a high rate of morbidity and mortality. Whereas cerebral malaria and other neurological sequelae have been described among under-fives and adults, a case of delayed cerebellar ataxia has not been published among adolescents in Uganda.

Case Presentation: A 10-year-old, HIV- negative girl who initially presented with severe headache and new-onset seizures, who developed severe cerebellar ataxia and vertigo on Day 14 after treatment. Notably, she had no other risk factors for cerebellar ataxia and had a normal cerebrospinal fluid and a normal CT-scan report. She was treated with anti-malarial medication, anti-convulsant therapy and antihistamines. She was discharged home in a stable condition.

Conclusion: We describe a case of delayed cerebellar ataxia in a 10 year-old HIV negative adolescent girl after completing her course of anti-malarial treatment. This case highlights the need of immediate follow-up of adolescents with cerebral malaria since they may present with delayed cerebellar ataxia.
\end{abstract}

Keywords: Cerebral Malaria; Delayed Cerebellar Ataxia; Adolescent

\section{Abbreviations}

DCA: Delayed Cerebral Atrophy; PNM:Post Malaria Neurological Syndrome; CSF: Cerebrospinal Fluid; RDT: Rapid Diagnostic Test

\section{Background}

Severe malaria can mimic many other diseases that are also common in malaria-endemic countries. Severe malaria is clinically characterized by confusion or drowsiness with extreme weakness (prostration). In addition, the following may develop: Cerebral malaria with generalized convulsions, pulmonary oedema, severe anemia, renal failure, hypoglycaemia, metabolic acidosis, circulatory collapse/shock, spontaneous bleeding and laboratory evidence of disseminated intravascular coagulation (DIC), and macroscopic hemoglobinuria hyperthermia. Serious complications can arise in Plasmodium falciparum infection and also rarely in Plasmodium vivax. They may sometimes develop suddenly over a span of time as short as $12-24 \mathrm{~h}$, and may lead to death if not treated promptly and adequately. In adolescents, convulsions due to malaria must be differentiated from acute bacterial meningitis and other causes of seizures such as electrolyte imbalance or metabolic insufficiency. 


\section{Case Report}

A 10-year-old adolescent girl was referred with complaints of 2 weeks history of headache, malaise, non-bilious vomiting and generalized tonic-clonic seizures which had lasted two days. Her symptoms were associated with generalized weakness, inability to sit or stand without support, slurred speech, and altered mentation but no loss of consciousness. Prior to referral to our health facility, she was treated for P. falciparum malaria with Artesunate and Arthemether- Lumefantrine without improvement. The physical examination on admission was normal with an exception of her CNS which showed a broad swaying gait with intention tremors and failed nose to finger test. There was also increased tone in both upper and lower limbs with brisk reflexes, however she had normal sensation. Her repeat blood tests were for positive malaria on a rapid diagnostic test (RDT), although the thin film was negative. The findings of a full blood count, liver function tests, renal function tests, and vitamin B12 and D levels were normal. Brain CT scan done reveal normal findings.

She was diagnosed with Severe Malaria and delayed cerebellar ataxia and managed with intravenous Artesunate at a dose of $240 \mathrm{mg} 8$ hourly and then tablets of athemether -lumefantrine. She also received $100 \mathrm{mg} / \mathrm{kg}$ of Ceftriaxone daily for 10 days as prophylaxis for bacterial sepsis. During her admission, various consultants including child psychiatry, physiotherapy; pediatric neurology and the rhino laryngology teams. In view of her excessive dizziness of the ENT made a diagnosis of Central vestibulopathy and she was started on antihistamine cinnarizine. Cinnarizine is an antihistamine and calcium channel blocker of the diphenylmethylpiperazine group. It is also known to promote cerebral blood flow, and so is used to treat cerebral apoplexy, post-trauma cerebral symptoms, and cerebral arteriosclerosis. However, it is more commonly prescribed for nausea and vomiting due to motion sickness or other sources such as chemotherapy, vertigo, or Ménière's disease [1]. She was discharged 23 days later with improvement in the CNS signs and she was to continue her physiotherapy from a nearby health facility.

\section{Discussion and Conclusion}

The patient's acute symptoms began 14 days after completion of her first course of antimalarial treatment. The patient was disoriented, intermittently awake, and obtunded but was arousable and unable to communicate. The neurological symptoms and signs lasted for 12 days, during which time no other new adverse events occurred [2]. The clinical manifestations of post malaria neurological syndrome (PMNS) in this case are consistent with the descriptions offered by Nguyen., et al. [3]. It is unlikely that the patient's symptoms were due to the toxic effect of antimalarial treatment, Vitamin B Complex or the antibiotics provided. She was treated with artesunate for 3 days, followed by a full course of coartem. The first description of PMNS appeared only recently, in 1996 [2]. Therefore, there is some speculation regarding whether PMNS is a true distinct clinical entity and not a consequence of malarial cerebral damage or neurologic sequel to antimalarial therapy [4,5]. In this case, we found evidence of cerebral malaria, and we administered antimalarials and anticonvulsants as well as antibiotics. We had another differential diagnosis of delayed cerebellar ataxia because of her typical presentations. Studies of a rare self-limiting postinfectious complication of malaria-delayed cerebellar ataxia (DCA), which was first identified in Sri Lanka in 1984-may provide clues to the etiology of the neuropathologic process responsible for post malaria neurological syndrome(PMNS) [6]. In DCA, the serum and CSF concentrations of certain cytokines, including TNF- $\alpha$, IL-2, and IL-6, are elevated, and a favorable response to corticosteroid therapy supports the possibility that an underlying immune mechanism is present. The selective focal cerebellar ataxia of DCA, however, is distinct from the diffuse constellation of symptoms seen in cases of PMNS, which supports a lower, more subtle degree of antibody cross-reactivity in patients with PMNS [6]. Idro., et al. in Uganda have described neurological manifestations among children discharge from hospital [7]. Cerebral malaria is the most severe neurological complication of falciparum malaria and a leading cause of death and neuro-disability in sub-Saharan Africa [7]. In a retrospective review by R. Idro., et al. they found that deficits in motor function, behaviour, vision, speech and hearing or epilepsy were major long-term sequelae. Two main patterns were observed: a) immediate and, b) late-onset deficits. Some deficits (e.g. blindness) resolved, others (e.g. loss of speech) showed little improvement over the follow-up period, while some (e.g. behaviour problems) developed long after exposure. Post malarial neurologic syndrome (PMNS) are known entities for decades. The important neurological sequelae (PMS) in survivors of cerebral malaria may include psychosis, cerebellar ataxia, hemiplegia, peripheral neuropathy and all show complete recovery on further follow-up. Delayed cerebellar ataxia is a new PMNS observed in the Ugandan context. The ataxic symptoms in our patient appeared after an afebrile period of 
14 days and neurological examination revealed no other abnormality except a cerebellar syndrome interfering with normal gait and speech. Among children followed up in India, the lower limbs were affected more than the upper limbs and the mean delay between the onset and fever and onset of cerebellar ataxia was 13 days [8].

To our knowledge, this patient's delayed cerebellar ataxia has not been described among adolescents presenting within 14 days of having cerebral malaria in our health facility. The patient had complete neurological recovery when she was discharged at Day 23 from the day of her admission. As the epidemiology of malaria changes particularly among older children and adolescents additional studies are needed to ascertain the epidemiology and pathogenesis of delayed cerebellar ataxia. The epidemiology and pathogenesis will only be revealed in high malaria prevalence areas and when we have a higher index of suspicion for DCA.

\section{Acknowledgements}

We would like the thank the patient and her caretaker for consenting to publication of her story.

\section{Authors' Contributions}

AWS and NS wrote the initial draft of the manuscript. SBK conceptualized the case report and edited the final draft. NM,VM and EAM edited the case report and read the final draft. SBK oversaw the project. All authors read and approved the final manuscript.

\section{Funding}

The authors have no funding to declare.

\section{Availability of Data and Materials}

Not applicable.

\section{Ethics Approval and Consent to Participate}

This case report received IRB approval from Mulago Hospital Ethics and Research Committee. We have obtained written consent from the primary caregiver of the child (the mother) and written assent from the child to publish the case report and associated images. These documents are available upon request.

\section{Consent for Publication}

We have obtained written consent from the primary caregiver of the child (the grandmother) and written assent from the child to publish the case report. These documents are available upon request.

\section{Competing Interests}

The authors declare that they have no competing interests.

\section{Bibliography}

1. Scholtz AW., et al. "Treatment of vertigo due to acute unilateral vestibular loss with a fixed combination of cinnarizine and dimenhydrinate: a double-blind, randomized, parallel-group clinical study". Clinical Therapeutics 26.6 (2004): 866-877.

2. Bajiya $\mathrm{H}$ and Kochar D. "Incidence and outcome of neurological sequelae in survivors of cerebral malaria". The Journal of the Association of Physicians of India 44.10 (1996): 679-681.

3. Mai NTH., et al. "Post-malaria neurological syndrome". The Lancet 348.9032 (1996): 917-21.

4. Dollow S. "Neurological sequelae of cerebral malaria". Lancet 348.9042 (1996).

5. Keusch GT. "The Year book of infectious diseases: Mosby" (1998).

6. Senanayake N and De Silva H. "Delayed cerebellar ataxia complicating falciparum malaria: a clinical study of 74 patients". Journal of Neurology 241.7 (1994): 456-459.

7. Idro R., et al. "Cerebral malaria is associated with long-term mental health disorders: a cross sectional survey of a longterm cohort”. Malaria journal 15.1 (2016): 184.

8. Kumar PS., et al. "Acute cerebellar ataxia: A neurological manifestation in malaria". Annals of Tropical Medicine and Public Health 7.6 (2014): 266.

\section{Volume 5 Issue 12 December 2021 (C) All rights are reserved by Edison Arwanire Mworozi., et al.}

